\title{
Assessment of the delayed repair of uncomplicated inguinal hernias in infants
}

\author{
SN Botes, ${ }^{1}$ J Edge, ${ }^{1}$ JP Apffelstaedt, ${ }^{2}$ D Sidler ${ }^{3}$ \\ ${ }^{1}$ Division of General Surgery, Department of Surgical Sciences, Faculty of Medicine and Health Sciences, Stellenbosch \\ University, South Africa \\ ${ }^{2}$ Panaroma, Private Practice, South Africa \\ ${ }^{3}$ Division of Paediatric Surgery, Tygerberg Children's Hospital, University of Stellenbosch, South Africa
}

Corresponding author, email: snbotes@gmail.com

Background: Potential strangulation of infant inguinal hernias is the main indication for their urgent repair. Lack of theatre time delays repair and prolongs hospitalisation. We report a series of patients with uncomplicated hernias who were discharged home to have their elective surgery at a later stage and assessed the outcomes of this approach.

Methods: A retrospective audit was performed of all infants with an inguinal hernia from January 2010 to June 2015. Incomplete records and infants operated after their first birthday were excluded. Two groups were identified; immediate surgery for infants with uncomplicated hernias, and delayed surgery for infants with uncomplicated hernias. Incarceration/ strangulation rates in the interim period were documented for the delayed group, and comparison made between the groups regarding perioperative and anaesthetic complications and length of postoperative hospital stay.

Results: The mean time delay between diagnosis and repair was 8.78 weeks. None of the hernias in the delay group strangulated while awaiting repair. There was no significant difference in the perioperative complications between the two groups. Out of the 70 cases in the immediate repair group, there was $7(10 \%)$ surgical and $4(5.7 \%)$ anaesthetic complications. The delayed group (169 infants) had 8 (4.7\%) surgical and $6(3.6 \%)$ anaesthetic complications. The incarceration rate after being discharged home was $4.1 \%$. This group of infants had no anaesthetic or surgical complications. Length of hospital stay postoperatively was 1.43 days in the immediate group and 1.3 in the delayed group $(p=.485)$.

Conclusion: Delayed repair, up to 2 months later, for uncomplicated infant hernia carries a small risk of incarceration but does not increase the rate of strangulation or other complications.

Keywords: infant, inguinal hernia, delayed surgery, incarceration, perioperative complications

\section{Introduction}

Inguinal hernias are common in infants, being reported to occur in up to $5 \%$ of newborns and $11 \%$ of infants born prematurely. ${ }^{1-3}$ Current accepted practice is to repair all uncomplicated inguinal hernias on the next available elective list. ${ }^{4-6}$ However, lack of theatre time and postoperative ICU beds delays the surgery and prolongs waiting time. ${ }^{7}$

Whether a delay between diagnosis and surgery is detrimental to the infant has been examined for inpatients, where management is guided by available resources. This would either be surgery on the next available elective list, or after an unwell infant is ready for discharge from tertiary care (ICU or ward) ${ }^{7,8}$ Less data is available for the outcome of infants with an uncomplicated hernia who have been sent home to be readmitted for a delayed repair, at a later stage. We present a large series from a developing country to identify patients in whom such an approach may be acceptable.

\section{Material and methods}

A retrospective audit was performed from records of all infants, who had an elective inguinal herniotomy during the period January 2010 to June 2015 at a tertiary institution of a developing country. Records were analysed for the following data: gestational age of the infant, birth weight, weight at surgery, age of the infant at surgery, gender, time in weeks between diagnosis and surgery, waiting time complications (incarceration or strangulation), surgical complications (intraoperative damage to adjacent structures or postoperative complications, such as recurrence, iatrogenic cryptorchidism and wound infection), anaesthetic complications (bradycardia, postoperative apnoea, pneumonia, laryngospasm and cardiac arrest), and average length of hospital stay (in days postoperative until fit for discharge from the surgical unit).

The following definitions were used: incarceration the need for sedation to reduce the hernia. ${ }^{9}$ Strangulation - vascular compromise of the hernia contents. ${ }^{4,5}$ Wound complications - superficial wound infection or haematoma formation. Damage to adjacent structures - vas deferens or gonadal artery as documented intraoperatively. Postoperative apnoea was defined as an apnoea episode witnessed by staff, detected on monitors longer than 15 seconds duration or if the infant desaturated to $<85 \% .^{10}$ 
Inclusion criteria were all hernias repaired in infants less than one year of age at Tygerberg Children's Academic Hospital between January 2010 and June 2015. Hernias diagnosed in infants less than 1 year, and then operated on after the infant's first birthday were excluded. Infants that presented with a complicated hernia previously undiagnosed or admitted for complicated medical conditions were also excluded. Cases with incomplete data were also excluded.

\section{Statistics}

Data were analysed separately for infants with uncomplicated inguinal hernias operated during first admission, and infants operated during second admission after having been discharged home to have surgery at a later stage. Subgroup analysis included infants that developed a complication while awaiting surgery.

All the data collected were entered into pre-designed Excel spreadsheets (Excel 2013, Microsoft Corporation, Redmond WA, USA). Statistical analysis was performed in Stata (StataCorp, College Station TX, USA) and Statistical Package for the Social Sciences. ${ }^{11}$ Data were reported as medians and interquartile ranges for continuous variables and frequencies and percentages for categorical variables. T-tests were conducted to determine whether any group differences existed as mentioned previously. A $p$-value of 0.05 was deemed significant.

\section{Results}

In the defined period, 677 infants were diagnosed with an inguinal hernia. Figure 1 is a flow diagram of our selection process. Eighty-four of these infants were lost to follow-up and excluded from the study. Three hundred and two cases had some data missing, mostly the time between booking and surgery, and so were also excluded. Of the remaining 291 cases, 169 patients (58\%) had uncomplicated inguinal hernias and were discharged home for delayed repair (Group 2). One hundred and twenty-two infants had surgery during same admission. Out of these 122 infants, 52 were excluded from the control group, due to being complicated on first presentation (prolonged hospital stay for medical reasons, or first presentation being an incarcerated/ strangulated hernia). This reduced our control group of uncomplicated hernias repaired at first presentation to 70 (Group 1). Table 1 summarises the age, gender and weight at surgery.

Comparing the perioperative complications between the immediate repair groups with the delayed group revealed no significant difference. The length of hospital stay was shorter in the delayed group, although this was not statistically significant. In the delayed group, 56 infants had bilateral hernias $(16.6 \%)$. The total number of hernias repaired in this group was 225 . Eleven infants developed an incarcerated hernia (4.9\%) while waiting, and no case of strangulation was recorded. When comparing these 11 cases to the uncomplicated group with immediate repair, there was no statistical significance in perioperative complications or length of hospital stay. The perioperative variables are summarised in Table 2. The average waiting period was 8.8 \pm 8.1 weeks. Some infants only waited 1 week, while the longest waiting time was 49 weeks.

Out of the 11 cases that developed a complication while awaiting surgery, no cases of surgical or anaesthetic complications were noted. The length of hospital stay in

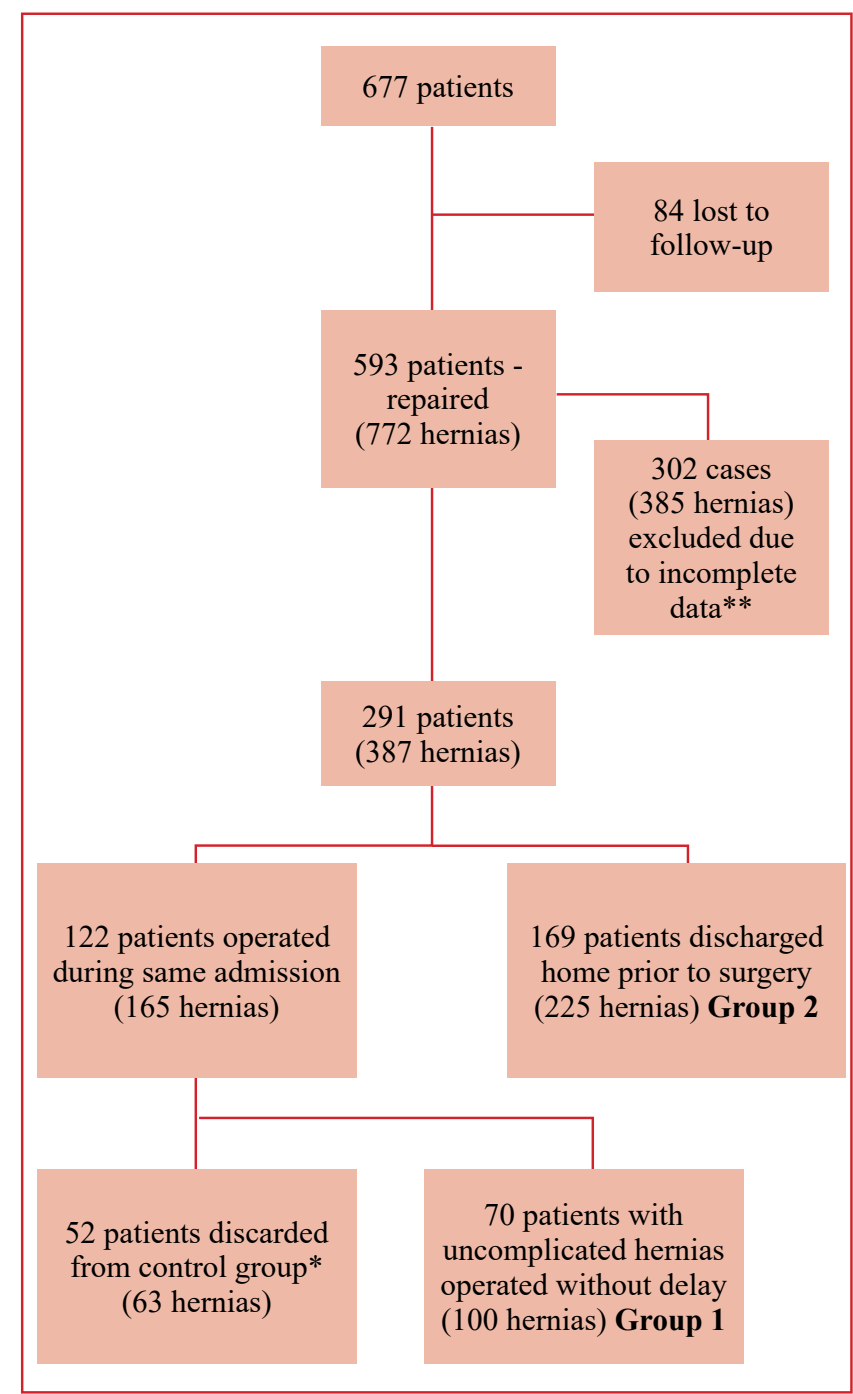

Figure 1

* 52 cases where discharged from the control group, because they had an incarcerated hernia as their first presentation. The hernia is therefore no longer uncomplicated and they were excluded.

** These 302 cases were discarded because some of the data was not complete. Mostly weight at surgery was not always clearly indicated, and any anaesthetic complications or lack thereof were not clearly stated. This group mostly consisted of infants sent home for surgery as a delayed procedure. It also included a group of infants where either no hernia was identified intra op, or where a hydrocele was diagnosed instead of a hernia.

these 11 cases were $1.45 \pm 1.03$. Five out of the 11 infants were preterm infants (Gestational age range between 31 and 33 weeks). The interval between diagnosis/discharge and presenting with incarceration was on average 8 weeks (median of 7.5) Compared to our control group of immediately repaired uncomplicated hernias, there was no statistical significance. See Table 3 for comparison.

We also compared the group of infants that were operated immediately but presented with a complication versus the uncomplicated immediate repair group. This group of infants had more anaesthetic complications $(p=0.014)$ and a longer postoperative hospital stay. Both anaesthetic and surgical complications were increased in this group, as seen in Table 4. The 3 surgical complications in this group that presented with complicated hernias were 2 anastomotic 
Table 1: Gender, gestational age and weight at surgery

\begin{tabular}{lccc}
\hline & $\begin{array}{c}\text { Group 1 } \\
(\boldsymbol{n}=\mathbf{7 0}) *\end{array}$ & $\begin{array}{c}\text { Group 2 } \\
(\boldsymbol{n}=\mathbf{1 6 9}) * *\end{array}$ & $\boldsymbol{P}$-value \\
\hline Male:Female & $9: 1$ & $9.2: 1$ & .560 \\
Premature $n(\%)$ & $45(64.3 \%)$ & $102(60.4 \%)$ & .598 \\
Weight $<3 \mathrm{~kg}$ at & $19(27.1 \%)$ & $18(10.8 \%)$ & .004 \\
surgery $n(\%)$ & & \\
*Group 1 - Uncomplicated hernia, repaired at initial hospitalisation & \\
$* *$ Group 2 - Uncomplicated hernia, delayed repaired &
\end{tabular}

Table 2: Perioperative variables

\begin{tabular}{lccc}
\hline & $\begin{array}{c}\text { Group 1 } \\
(\boldsymbol{n}=\mathbf{7 0})\end{array}$ & $\begin{array}{c}\text { Group 2 } \\
(\boldsymbol{n}=\mathbf{1 6 9})\end{array}$ & $\boldsymbol{P}$-value \\
\hline $\begin{array}{l}\text { Surgical } \\
\text { complications } n(\%)\end{array}$ & $7(10 \%)$ & $8(4.7 \%)$ & .454 \\
$\begin{array}{l}\text { Anaesthetic } \\
\text { complications } n(\%)\end{array}$ & $4(5.7 \%)$ & $6(3.6 \%)$ & .343 \\
$\begin{array}{l}\text { Length of stay postop } \\
\text { days Mean } \pm \text { SD }\end{array}$ & $\begin{array}{c}1.43 \pm \\
1.48\end{array}$ & $1.3 \pm 1.271$ & .485 \\
\end{tabular}

Table 3: Subgroup comparison of incarcerated hernias in delayed surgery group

\begin{tabular}{|c|c|c|c|}
\hline & $\begin{array}{l}\text { Group 1 } \\
(n=70)\end{array}$ & $\begin{array}{c}\text { Waiting period } \\
\text { complications }(n=11)\end{array}$ & $P$-value \\
\hline $\begin{array}{l}\text { Surgical } \\
\text { complications } \\
n(\%)\end{array}$ & $7(10 \%)$ & 0 & .377 \\
\hline $\begin{array}{l}\text { Anaesthetic } \\
\text { complications } \\
n(\%)\end{array}$ & $4(5.7 \%)$ & 0 & .466 \\
\hline $\begin{array}{l}\text { Length of stay in } \\
\text { days Mean } \pm \text { SD }\end{array}$ & $\begin{array}{c}1.43 \pm \\
1.48\end{array}$ & $1.45 \pm 1.03$ & .956 \\
\hline
\end{tabular}

Table 4: Comparison of infants presenting with complications vs control group

\begin{tabular}{lccc}
\hline & $\begin{array}{l}\text { Group 1 } \\
(\boldsymbol{n}=\mathbf{7 0})\end{array}$ & $\begin{array}{c}\text { Complication as first } \\
\text { presentation }(\boldsymbol{n}=\mathbf{5 2})\end{array}$ & $\boldsymbol{P}$-value \\
\hline $\begin{array}{l}\text { Surgical } \\
\text { complications } \\
n(\%)\end{array}$ & $7(10 \%)$ & $3(5.7 \%)$ & .083 \\
$\begin{array}{l}\text { Anaesthetic } \\
\text { complications } \\
n(\%)\end{array}$ & $4(5.7 \%)$ & $6(11.5 \%)$ & .014 \\
$\begin{array}{l}\text { Length of stay in } \\
\text { days Mean } \pm \text { SD }\end{array}$ & $\begin{array}{c}1.43 \pm \\
1.48\end{array}$ & $2.75 \pm 4.756$ & .375 \\
\end{tabular}

leaks following resection, and one infant had a missed Ileal perforation.

Eighty-four patients were lost to follow-up, after being booked for delayed hernia repair. In 54 of these patients $(64 \%)$ there is no proof that they were seen at Tygerberg Children's Hospital after discharge. Twenty-three (27\%) patients did not arrive for the elective operation date. At a later visit, to a different division at Tygerberg Children's Hospital, no mention was made of the inguinal hernia. Four patients $(4.7 \%)$ initially missed the follow-up date, only to have the surgery after their first birthday (and hence were excluded from the study). No complications were recorded during this waiting period. Two patients had no hernia detectable on admission, and one parent arrived on the elective day, only to inform us that they no longer wanted surgery.

\section{Discussion}

In a survey conducted by The American Academy of Paediatrics (AAP), two thirds of paediatric surgeons would repair an inguinal hernia before discharge, if diagnosed during initial admission; if the hernia was only diagnosed after discharge, approximately half of surgeons would repair it immediately. ${ }^{8}$ The reason for early elective repair is the perceived risk of incarceration or strangulation. This risk is often quoted as high as $30 \%$ in infants. Yet some of the initial studies that determined this risk were small and are now outdated. ${ }^{1,2}$ In a more recent series, Sulkowski et al. reviewed over 2000 patients that either had delayed or immediate repair and reported an incidence of only $9.5 \%$ incarceration in the waiting period for delayed hernia repair. Their series was conducted in a first world country, however, and the time delay between diagnosis and surgery was not specified. ${ }^{9}$

The diagnosis of incarceration is usually made at first point of contact at a primary health clinic. This diagnosis is often incorrect and could lead to overestimation of the true incidence of incarceration. When applying the definition of incarceration, that sedation is needed in order to manually reduce the hernia, the incidence is closer to $10 \%$ compared to the $30 \%$ originally quoted..$^{9,12-14}$ In our series, the risk was still much lower. Eleven infants out of the 169 infants who were discharged prior to repair presented with incarceration $(6.5 \%)$. The number of infants with bilateral inguinal hernias needs to be considered, seeing as how both inguinal hernias are at risk of complicating. Our rate of incarceration was therefore 11 out of 225 hernias, an incarceration rate of only $4.9 \%$. Also worth noting is the low incidence of perioperative complications in this group of patients. From our results it appears that incarceration does not result in more anaesthetic or surgical complications, and the length of hospital stay is not adversely affected in this group.

The characteristics of our patients are in keeping with current literature. The incidence was higher in males, and the right side more commonly affected than the left. A significant number $(60.2 \%)$ of inguinal hernias occurred in preterm infants. ${ }^{4-6}$ Baird et al. ${ }^{12}$ determined that prematurity was a more important risk factor in inguinal hernia complication rate compared with age at surgery. That study was, however, aimed more at the postoperative complication rate rather than

Table 5: Comparison between similar series

\begin{tabular}{lcccc}
\hline & Crankson & Lee & Sulkowski & Current series \\
\hline Total cases in series & 84 & 172 & 2030 & 350 \\
Delayed repair group $n(\%)$ & $61(72.6 \%)$ & $127(73.8 \%)$ & $667(32.9 \%)$ & $225(64.3 \%)$ \\
Incarceration $n(\%)$ & $2(3.20 \%)$ & 0 & $55(8.20 \%)$ & $11(4.9 \%)$ \\
Immediate repair group $n(\%)$ & $23(27.4 \%)$ & $45(26.2 \%)$ & $1363(67.1 \%)$ & $125(35.7 \%)$ \\
Incarceration $n(\%)$ & 0 & $8(17.8 \%)$ & $161(11.80 \%)$ & $51(41 \%)$
\end{tabular}


the waiting period or intraoperative complications. Hughes et al. ${ }^{15}$ found that preterm infants had a significantly higher rate of incarceration. This was attributed to a smaller hernia defect, co-existing lung disease and the need for positive pressure ventilation. Their study was also, however, aimed at the complication rate following surgical repair as opposed to the waiting period complication. Our series with patient characteristics similar to those in resource rich environments and with a very low complication rate demonstrates that our approach to discharge patients with uncomplicated hernias for repair on an elective list, up to 2 months later, is safe and alleviates pressure on emergency lists.

The 84 patients that did not arrive for the corrective surgery also needs some discussion as this could be viewed as a complication. We acknowledge the fact that some of these cases could have incarcerated. In our referral system however, all incarcerated hernias will be referred back to us, unless they moved into a different drainage area. A number of patients were seen however at Tygerberg hospital subsequently with no evidence of complication and in some cases no evidence of a hernia anymore. An argument can therefore be made that the cases that missed the elective appointment are not necessarily a complication of delaying uncomplicated hernias.

Table 5 compares this series with similar studies done in developed countries. Crankson and co-workers' series concluded that delaying repair in preterm infants until they were ready to be discharged from the neonatal intensive care unit is safe. They also reported a higher rate of recurrence in the group where surgery was performed earlier. They did not conclude that it is safe to discharge patients from hospital prior to inguinal hernia repair, although the incarceration rate was only $3.2 \%$ in that group. ${ }^{16}$ In the group of preterm infants, who were discharged for elective herniotomy in Lee's series, none of the 127 patients suffered an incarceration. ${ }^{17}$ Sulkowski's series included a significant number of infants who were discharged with a known inguinal hernia for delayed repair. Of note was that the infants selected for delayed repair had more congenital abnormalities, required prolonged ventilation and TPN for other medical conditions prior to surgery. The 30-day emergency department visit, mortality rate and inguinal hernia related complications were similar for both groups. The early group, however, had longer hospital stay, required transfusion more frequently and had a higher likelihood of requiring a second operation $(8.1 \%$ vs $3.2 \%){ }^{9}$

\section{Conclusion}

This series demonstrates that, in a resource limited country, it is safe to discharge an infant with uncomplicated inguinal hernia home. Smaller infants have technically more challenging surgery and potentially higher anaesthetic complication rate. They also have a longer hospital stay. Incarceration of inguinal hernia while awaiting elective repair was not as common as anticipated, and none of these infants suffered higher surgical or anaesthetic complications than the group who were repaired immediately. It would be worthwhile to undertake a prospective trial to answer the relevant question. Is it imperative to repair all uncomplicated inguinal herniae immediately, or is there a safe amount of time that one can wait, allowing the infant to gain weight and be operated on an elective list?

\section{Conflict of interest}

We wish to confirm that there are no known conflicts of interest associated with this publication and there has been no significant financial support for this work that could have influenced its outcome.

\section{Compliance with ethical standards}

Ethical approval: The research protocol was approved by the Health Research and Ethics Committee of the University of Stellenbosch. Protocol no. S16/10/218.

All procedures performed in studies involving human participants were in accordance with the ethical standards of the institutional research committee and with the 1964 Helsinki declaration and its later amendments or comparable ethical standards.

\section{REFERENCES}

1. Harper RC, Garper A, Sia C. Inguinal Hernia : A Common Problem of Premature Infants Weighing or Less at Birth. Pediatrics. 1975;56(1):112-5.

2. Peevy KJ, Speed FA, Hoff CJ. Epidemiology of inguinal hernia in preterm neonates. Pediatrics. 1986;77:246-7.

3. Stylianos S, Jacir N, Harris B. Incarceration of inguinal hernia in infants prior to elective repair. J Paediatr Surg. 1993;28(4):582-3.

4. Puri P. Newborn Surgery. 2nd ed. Hodder Arnold; 2003. p5618.

5. Holcomb GW, Murphy JP. Aschraft's Paediatric Surgery. 5th edition. Saunders Elsevier; 2010. 669-75.

6. Ameh E, Bickler S, Lakhoo K, et al. Paediatric surgery: A comprehensive text for Africa. Global HELP; 2012. 358-65.

7. Chen LE, Zamakhshary M, Foglia RP, et al. Impact of wait time on outcome for inguinal hernia repair in infants. Pediatr Surg Int. 2009;25:223-7.

8. Antonoff MB, Kreykes NS, Saltzman DA, et al. American Academy of Pediatrics section on surgery hernia revisited. J Pediatr Surg. 2005;40:1009-14.

9. Sulkowski JP, Cooper JN, Duggan EM, et al. Does timing of neonatal inguinal hernia repair affect outcomes? J Pediatr Surg. 2014;50:171-6.

10. Laituri CA, Garey CL, Pieters BJ, Mestad P, Weissend EE, St Peter SD. Overnight observation in former premature infants undergoing inguinal hernia repair. J Pediatr Surg. 2012;47:217-20.

11. IBM Corp. IBM SPSS Statistics for Macintosh, Version 24.0. 2016.

12. Baird R, Gholoum S, Laberge JM, Puligandla P. Prematurity, not age at operation or incarceration, impacts complication rates of inguinal hernia repair. J Pediatr Surg. 2011;46:908-11.

13. Gholoum S, Baird R, Laberge JM, Puligandla PS. Incarceration rates in pediatric inguinal hernia: do not trust the coding. J Pediatr Surg. 2010;45:1007-11.

14. Lautz TB, Raval MV, Reynolds M. Does timing matter? A national perspective on the risk of incarceration in premature neonates with inguinal hernia. J Pediatr. 2011;158:573-7.

15. Hughes K, Horwood JF, Clements C, et al. Complications of inguinal herniotomy are comparable in term and premature infants. Hernia. 2016;20:565-9.

16. Crankson SJ, Al Tawil K, Al Namshan M, Al Jadaan S, Baylon BJ, Gieballa M, et al. Management of inguinal hernia in premature infants: 10-year experience. J Indian Assoc Pediatr Surg. 2015;20:21-4.

17. Lee SL, Gleason JM. A review of prem infants with inguinal hernias: Optimal timing of repair, incarceration risk, and postop apnea. J Ped Surg. 2011;46:217-20. 